12. Foretz M, et al. Metformin inhibits hepatic gluconeogenesis in mice independently of the LKB1/ AMPK pathway via a decrease in hepatic energy state. J Clin Invest. 2010;120(7):2355-2369.

13. Berglund ED, et al. Hepatic energy state is regulated by glucagon receptor signaling in mice. J Clin Invest. 2009;119(8):2412-2422.

14. Tanaka T, Sue F, Morimura H. Feed-forward activation and feed-back inhibition of pyruvate kinase type L of rat liver. Biochem Biophys Res Commun. 1967; 29(3):444-449.

15. Kemp RG. Rabbit liver phosphofructokinase. Comparison of some properties with those of muscle phosphofructokinase. J Biol Chem. 1971; 246(1):245-252.

16. Van Schaftingen E, Hers HG. Inhibition of fructose1,6-bisphosphatase by fructose 2,6-biphosphate. Proc Natl Acad Sci U S A. 1981;78(5):2861-2863.

17. Taylor SI, Mukherjee C, Jungas RL. Regulation of pyruvate dehydrogenase in isolated rat liver mitochondria. Effects of octanoate, oxidationreduction state, and adenosine triphosphate to adenosine diphosphate ratio. J Biol Chem. 1975; 250(6):2028-2035.

18. Burgess SC, et al. Cytosolic phosphoenolpyruvate carboxykinase does not solely control the rate of hepatic gluconeogenesis in the intact mouse liver. Cell Metab. 2007;5(4):313-320.

19. Samuel VT, et al. Fasting hyperglycemia is not associated with increased expression of PEPCK or G6Pc in patients with type 2 diabetes. Proc Natl Acad Sci US A. 2009;106(29):12121-12126

20. Shaw RJ, et al. The kinase LKB1 mediates glucose homeostasis in liver and therapeutic effects of metformin. Science. 2005;310(5754):1642-1646.

21 . He L, et al. Metformin and insulin suppress hepatic gluconeogenesis through phosphorylation of CREB binding protein. Cell.
2009;137(4):635-646.

22. Mu J, Brozinick JT Jr, Valladares O, Bucan M, Birnbaum MJ. A role for AMP-activated protein kinase in contraction- and hypoxia-regulated glucose transport in skeletal muscle. Mol Cell. 2001;7(5):1085-1094.

23. Musi N, Hayashi T, Fujii N, Hirshman MF, Witters LA, Goodyear LJ. AMP-activated protein kinase activity and glucose uptake in rat skeletal muscle. Am J Physiol Endocrinol Metab. 2001; 280(5):E677-E684.

24. Kalender A, et al. Metformin, independent of AMPK, inhibits mTORC1 in a rag GTPase-dependent manner. Cell Metab. 2010;11(5):390-401.

25. Libby G, Donnelly LA, Donnan PT, Alessi DR, Morris AD, Evans JM. New users of metformin are at low risk of incident cancer: a cohort study among people with type 2 diabetes. Diabetes Care. 2009;32(9):1620-1625.

\title{
Radioprotection: smart games with death
}

\author{
Andrei V. Gudkov1,2 and Elena A. Komarova ${ }^{1}$
}

1Department of Cell Stress Biology, Roswell Park Cancer Institute, and ²Cleveland BioLabs Inc., Buffalo, New York, USA.

\begin{abstract}
The efficacy of cancer treatment by radiation and chemotherapeutic drugs is often limited by severe side effects that primarily affect the hematopoietic system and the epithelium of the gastrointestinal tract. Progress in understanding differences in the mechanisms involved in the responses of normal and tumor cells to genotoxic stress has led to the development of new rational approaches to selective protection of normal cells, such as suppression of apoptosis by pharmacological inhibition of p53 or activation of NF- $\kappa B$. Another promising approach presented in this issue by Johnson et al. is based on the idea of using pharmacological inhibitors of cyclin-dependent kinases (CDKs) to convert normal cells into a radioresistant state by inducing reversible cell cycle arrest at the $G_{1} / S$ transition. The evidence indicates that this approach is likely to be specific for protection of normal cells and may, therefore, have clinical potential as an adjuvant in anticancer therapies.
\end{abstract}

\section{Importance and challenges of clinical radioprotection}

While radiotherapy is a powerful anticancer treatment approach, it is associated with severe side effects that drastically limit its therapeutic capacity. Two of the most radiosensitive tissues - the hematopoietic (HP) system and the gastrointestinal (GI) tract - provide the biggest clinical challenges. The outcome of radiotherapy would be substantially improved if its therapeutic index could be increased by selective reduction of damage to the HP and GI systems, without an increase in the radioresistance of tumors. There is evidence indicating that

Conflict of interest: Andrei V. Gudkov is a shareholder in and consultant for Cleveland BioLabs Inc.

Citation for this article: J Clin Invest. 2010; 120(7):2270-2273. doi:10.1172/JCI43794. increasing the cumulative radiation dose by as little as $10 \%-20 \%$ might make a difference between incomplete and complete eradication of tumors, as shown, for example, for head and neck cancer (1). In order to allow for increased radiotherapy doses, pharmacological approaches to radioprotection of normal tissues must be developed. These approaches must be strictly selective for healthy tissues and must be safe (i.e., must not promote the development of radiation-induced secondary cancers).

Since the killing of cells by radiation is mediated by the ionization of irradiated matter, the most well-characterized principle of radioprotection involves using antioxidants, which act as scavengers of reactive oxygen species. The only drug currently approved for clinical use to protect against the toxicity of radiotherapy, amifostine, works in this way (2). However, clinical use of amifostine is complicated by its own toxicity and lack of sufficient selectivity in protection of normal versus tumor cells (2).

\section{Targeting apoptotic mechanisms for radioprotection: p53 inhibitors}

In the 1990s, it became clear that the massive cell loss that occurs in radiosensitive tissues and embryos after irradiation and leads to lethality is not due to irreversible damage of cells but rather to activation of apoptosis. This apoptosis is largely p53 dependent, and p53-deficient mice are resistant to doses of radiation that kill wild-type mice by inducing lethal HP acute radiation syndrome (3). Importantly, apoptotic mechanisms are frequently inactivated in tumors, as part of their progression toward unconstrained growth (4). These findings presented an attractive opportunity for the development of pharmacological inhibitors of p53 capable of providing reversible radioprotection by temporarily blocking p53-mediated apoptosis. The feasibility of this idea was proven by isolation of small molecule p53 inhibitors, named pifithrins, that demonstrated radioprotective efficacy in mice $(5,6)$. Since p53-dependent apoptosis is considered a major component of p53 tumor suppressor activity, concerns regarding the safety of pharmacological p53 inhibition have slowed down clinical developments based on this approach. 


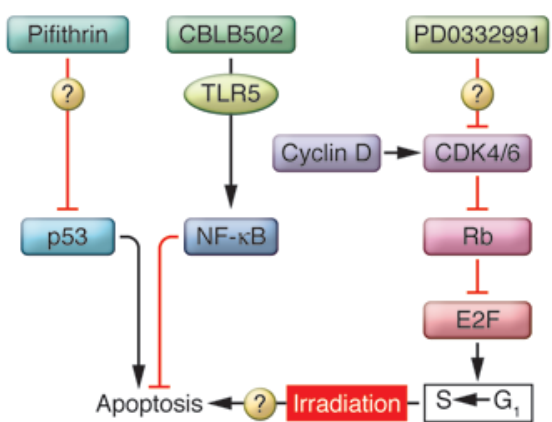

However, it has now been well documented, using both pharmacologic (7) and genetic (8) approaches, that temporary and reversible suppression of p53, resulting in massive rescue of cells in radiosensitive tissues, is not associated with an increase in carcinogenicity. Thus, p53 inhibitors are expected to emerge as tissue protective drugs to be used under pathological conditions associated with massive apoptotic cell death (9).

Although p53 inhibitors hold therapeutic promise, their potential application is limited by the tissue specificity of p53dependent radiosensitivity. Specifically, despite the pronounced p53-dependent apoptosis that occurs among epithelial cells of the small intestine after irradiation, clinical GI acute radiation syndrome develops independently of p53. Actually, p53-deficient mice are more susceptible to GI radiation damage than wild-type mice (10). Further, p53 acts as a survival factor in irradiated endothelial cells (11), and radiation-induced ceramide-mediated apoptosis of endothelial cells in the small intestine has been defined as a major effector of GI radiosensitivity in mice (12). These findings made it clear that radioprotection of the GI tract would require p53independent strategies.

\section{An alternative approach to block apoptosis: radioprotection via NF-KB activation}

A promising solution to this problem was found with another class of apoptosis inhibitors that mimic a different tumorspecific mechanism of cell survival: activation of the prosurvival NF- $\mathrm{BB}$ pathway. Being a major mediator of immune responses, NF-KB is generally inactive in healthy cells under normal conditions, but it is constitutively activated in most tumors, presumably as part of their sur-

\section{Figure 1}

Hypothetical mechanisms underlying three approaches to pharmacological protection of normal cells from ionizing radiation. All the mechanisms depicted are based on the presumption that apoptotic cell loss is a central event in both the HP and GI components of acute radiation syndrome. Small molecules known as pifithrins (5), acting through unknown mechanisms, inhibit p53-mediated apoptosis, thereby rescuing HP progenitors. The flagellin derivative CBLB502 activates NF-кB by inducing TLR5 signaling, also leading to suppression of apoptosis. Unlike p53 inhibitors, CBLB502 is effective not only against the HP but also the GI component of acute radiation syndrome (15). Constitutive activation of CDK4/6 frequently occurs in tumors (e.g., by overexpression of cyclin $\mathrm{D}$ ), leading to hyperphosphorylation of the tumor suppressor protein $\mathrm{Rb}$, blocking its ability to repress the E2F family of transcription factors that are essential for cell progression from the $\mathrm{G}_{1}$ to $\mathrm{S}$ phase of the cell cycle. As shown in this issue by Johnson et al. (16), the synthetic small molecule PD0332991, a specific CDK4/6 inhibitor with unknown mechanism of action, protects the HP system from radiation damage, presumably by inducing $\mathrm{Rb}$-mediated quiescence associated with suppression of apoptosis.

vival strategy (13). Among other targets, $\mathrm{NF}-\kappa \mathrm{B}$ drives expression of inflammatory cytokines, which have long been recognized to have radioprotective power (14); however, unacceptable toxicity has prevented their clinical development as radioprotectants. Recently, however, an NF- $\mathrm{KB}$-activating approach to radioprotection has been proven feasible by the demonstration that bacterial flagellin, which is an agonist of TLR5 and a natural NF-кB-activating agent, has outstanding radioprotective properties in mice and primates (15). Pharmacologic, constitutive engagement of NF- $\kappa \mathrm{B}$ activity is highly specific for protection of normal cells in mouse tumor models (15). Like p53 inhibitors, use of NF- $\mathrm{\kappa B}$ activators in combination with radiation is safe, in terms of lack of stimulation of radiation-induced carcinogenicity (15).

Both p53 inhibitors and NF- $\mathrm{KB}$ activators are currently in clinical development as tissue protectants for a variety of applications (Cleveland BioLabs Inc., www.cbiolabs.com/scientific_foundation_technol.php and Quark Pharmaceuticals, http://www.quarkpharma.com/ qbi-en/products/qpi-1002/). Another radioprotection concept, described in this issue by Johnson et al. (16), may open a new avenue for the development of radioprotectants. This concept is based upon the use of an emerging class of anticancer agents, inhibitors of cyclin-dependent kinases (CDKs), to target the cell cycle control machinery (Figure 1).

\section{Quiescence as a safe harbor: radioprotection via reversible cell cycle arrest}

The cytotoxicity of DNA-damaging agents, such as ionizing radiation, is cell cycle dependent (17). Cells are generally most sensitive at mitosis, when the chro- matin is subject to strong torsional stresses and when DNA breaks have almost no chance of being repaired before chromosome segregation. Cells in early $G_{1}$ and late $S$ phase are relatively radioresistant, whereas the $G_{1} / S$ transition and $G_{2} / M$ phase are relatively radiosensitive. These observations provided the rationale for Johnson et al. (16) to test cell cycle-targeting agents as potential radioprotectants (Figure 1).

The inhibitor of kinase 4/alternative reading frame (INK4a/ARF) locus encodes proteins that act as negative regulators of the cell cycle by inhibiting particular CDKs to cause cell cycle arrest at specific points of the cell cycle. Analysis of human malignancies has shown that the CDK-cyclin D/INK4/pRb/E2F pathway is abnormal in most cancers. Deregulation of CDK4/6 activity, either through overexpression of D-type cyclins or through loss of INK4 proteins, almost invariably leads to hyperproliferation and eventually to tumor development (18). Given their critical role in cell cycle control, CDKs have been actively considered as targets for anticancer therapy. A number of CDK inhibitors (CDKIs), with different mechanisms of action targeting regulatory pathways implicated in CDK function, have been evaluated in laboratory experiments and in human trials (19).

Johnson et al. (16) tested the effect of previously identified CDKIs that control the transition from $G_{1}$ to $S$ phase in ionizing radiation-induced cell toxicity. They confirmed that inhibitors specific for CDK4/6, specifically PD0332991 and 2BrIC, caused reversible $G_{1}$ arrest (pharmacological quiescence) exclusively in Rb-positive and not Rb-deficient human cells. In mice, oral administration of PD0332991 resulted in reversible inhibition of proliferation of different populations of bone marrow cells 


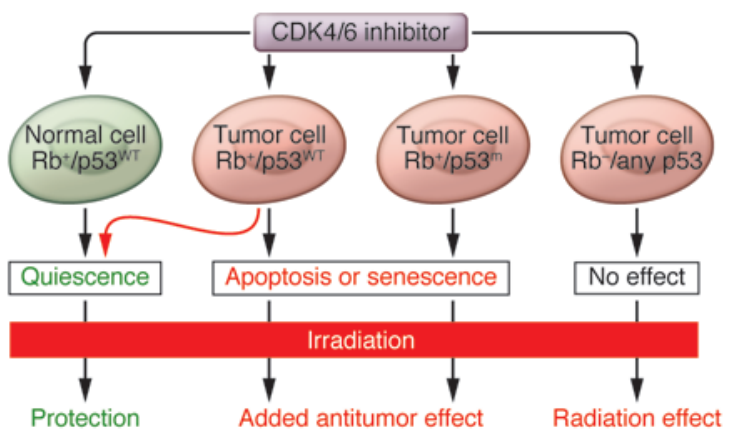

Figure 2

Radioprotection by CDK4/6 inhibitors is expected to be highly specific for normal cells. Normal cells respond to CDK4/6 pharmacological inhibition by reversible cell cycle arrest (quiescence). Lack of functional $\mathrm{Rb}$ makes cells insensitive to CDK4/6 inhibition, regardless of other genetic alterations. However, even those tumor cells that retain wild-type $\mathrm{Rb}$ may not be protected by CDK4/6 inhibitors, since they frequently have mechanisms of quiescence deregulated and respond to CDK4/6 inhibition by apoptosis or irreversible arrest (senescence; ref. 21). The irreversibility of CDK4/6 effects on Rb-positive tumor cells explains the efficacy of CDK4/6 inhibitors as antitumor agents. Only rare tumors retaining functional $\mathrm{Rb}$ and $\mathrm{p} 53$ and the capacity to respond to CDK4/6 inhibition by quiescence can be protected (red arrow). m, mutant.

(16). The least differentiated cells (HP stem cells, multipotent progenitor cells, and common myeloid progenitors) appeared to be more dependent on CDK4/6 than did the more differentiated bone marrow elements (granulocyte-monocyte progenitors and megakaryocyte-erythroid progenitors). Consistent with earlier reports (20), cells at the final stages of differentiation were found to be resistant to CDK4/6 inhibition (16).

The PD0332991-induced quiescence of bone marrow progenitor cells observed by Johnson and colleagues was accompanied by radioresistance (16). When administered to mice 4 hours before exposure to a radiation dose sufficient to induce lethal HP syndrome, PD0332991 protected the mice from death. In this scenario, PD0332991 had a beneficial effect on the recovery of all peripheral blood lineages: platelets, erythrocytes, myeloid cells, and peripheral blood lymphocytes. Moreover, no evidence of myeloproliferative disorder or myelodysplasia was found in animals of long-term surviving cohorts. These data indicate that the mechanism of PD0332991-mediated radioprotection is apparently safe and does not exacerbate the late hematological toxicity associated with high-dose total body irradiation.

\section{Why do CDK4/6 inhibitors protect normal cells but not tumor cells?}

It is expected that CDK4/6 inhibitors should not protect Rb-deficient cells, as they are CDK4/6 independent. Consistent with this, Johnson et al. showed that
PD0332991 did not protect CDK4/6-independent tumors against radiotherapy by studying mice that develop autochthonous melanomas, due to melanocyte-specific expression of mutant H-Ras (16). However, this does not mean that radioprotection by CDK4/6 inhibitors should be limited to patients with Rb-deficient tumors. On the contrary, these agents were originally considered as prospective anticancer drugs against tumors "addicted" to the high Rbsuppressive activity of CDK4/6 (Figure 1), a common property of a large proportion of tumors (19). As opposed to normal cells, in which pharmacological inhibition of CDK4/6 results in reversible quiescence, presumably converting them into radioresistant state, the response of tumor cells to these agents is frequently irreversible and involves the induction of senescence or apoptosis (Figure 2). It has been demonstrated that temporary overexpression of the natural CDK2 inhibitor p21 resulted in establishment of senescence in some human tumor cell lines, while cell cycle arrest remained reversible in normal diploid fibroblasts (21). The choice between senescence and quiescence in response to CDKI treatment is determined in part by p53 activity, specifically, the ability of p53 to serve as an inhibitor of the mTOR pathway (21). Hence, tumor cell radioprotection by PD0332991 or functionally similar compounds is expected to occur only in a minor subset of tumors that retain both $\mathrm{Rb}$ and p53 function, including the ability of p53 to negatively regulate mTOR (Figure 2).

\section{Puzzles and perspectives}

Johnson et al. interpret the radioprotection provided by CDK4/ 6 inhibitors as a function of cellular quiescence. This is a sound hypothesis but is currently supported only by correlative evidence. Since HP cell death following irradiation occurs primarily through apoptosis, this hypothesis suggests a link between quiescence and resistance to apoptosis, something that has not been previously reported for bone marrow HP precursor cells and that is definitely not true for thymocytes, splenocytes, and other radiosensitive mature HP cells. Hence, the exact mechanisms translating quiescence into radioresistance of HP cells remain to be elucidated.

Johnson et al. found that PD0332991 markedly enhanced the survival of mice, when applied as late as 20 hours after total body irradiation (16); thus, it acted as a radiomitigator. If this rescuing effect is indeed explained by drug-induced quiescence, it would suggest that premature entrance of HP progenitors into the cell cycle following DNA damage could be a lethal event for irradiated HP progenitors and contribute to HP acute radiation syndrome. This is a testable hypothesis that remains to be verified. Other possible explanations for PD0332991-mediated radiomitigation include involvement of direct and indirect effects of the compound on HP progenitors or bone marrow stroma.

Another unknown is the tissue specificity of the reported effects. If PD0332991 is found to be protective/mitigative not only against the HP component of acute radiation syndrome but also against the higher doses of ionizing radiation that induce severe GI damage, the potential clinical impact of CDK4/6 inhibitors would be greatly enhanced, with a broader scope of potential applications as radiotherapy and chemotherapy adjuvants as well as biodefense drugs.

\section{Acknowledgments}

We thank Patricia Stanhope-Baker for help on manuscript preparation. The authors' work on radioprotection is supported by grants CA075179 and AI080446 from NIH and from Cleveland BioLabs Inc. (to A.V. Gudkov).

Address correspondence to: Andrei Gudkov, Roswell Park Cancer Institute, Elm and Carlton St., Buffalo, New York 14263, USA. Phone: 716.845.3902; Fax: 716.845.3944; E-mail: andrei.gudkov@roswellpark.org. 
1. Bhide SA, Nutting CM. Advances in radiotherapy for head and neck cancer [published online ahead of print April 19, 2010]. Oral Oncol. doi:10.1016/ j.oraloncology.2010.03.005.

2. Weiss JF, Landauer MR. Protection against ionizing radiation by antioxidant nutrients and phytochemicals. Toxicology. 2003;189(1-2):1-20.

3. Komarova EA, et al. Transgenic mice with p53responsive lacZ: p53 activity varies dramatically during normal development and determines radiation and drug sensitivity in vivo. $E M B O J$. 1997;16(6):1391-1400.

4. Vousden KH, Prives C. Blinded by the Light: The Growing Complexity of p53. Cell. 2009;137(3):413-431

5. Komarov PG, et al. A chemical inhibitor of p53 that protects mice from the side effects of cancer therapy. Science. 1999;285(5434):1733-1737.

6. Strom E, et al. Small-molecule inhibitor of p53 binding to mitochondria protects mice from gamma radiation. Nat Chem Biol. 2006;2(9):474-479.

7. Leonova KI, et al. A small molecule inhibitor of p53 stimulates amplification of hematopoietic stem cells but does not promote tumor development in mice. Cell Cycle. 2010;9(7):1434-1443.

8. Christophorou MA, Ringshausen I, Finch AJ, Swigart
LB, Evan GI. The pathological response to DNA damage does not contribute to p53-mediated tumour suppression. Nature. 2006;443(7108):214-217.

9. Gudkov AV, Komarova EA. Prospective therapeutic applications of 553 inhibitors. Biochem Biophys Res Commun. 2005;331(3):726-736.

10. Komarova EA, et al. Dual effect of p53 on radiation sensitivity in vivo: $\mathrm{p} 53$ promotes hematopoietic injury, but protects from gastro-intestinal syndrome in mice. Oncogene. 2004;23(19):3265-3271.

11. Burdelya LG, et al. Inhibition of p53 response in tumor stroma improves efficacy of anticancer treatment by increasing antiangiogenic effects of chemotherapy and radiotherapy in mice. Cancer Res. 2006;66(19):9356-9361.

12. Paris F, et al. Endothelial apoptosis as the primary lesion initiating intestinal radiation damage in mice. Science. 2001;293(5528):293-297.

13. Karin M. Nuclear factor-kappaB in cancer development and progression. Nature. 2006; 441(7092):431-436.

14. Herodin F, Bourin P, Mayol JF, Lataillade JJ, Drouet M. Short-term injection of antiapoptotic cytokine combinations soon after lethal gamma -irradiation promotes survival. Blood. 2003;
101(7):2609-2616.

15. Burdelya LG, et al. An agonist of toll-like receptor 5 has radioprotective activity in mouse and primate models. Science. 2008;320(5873):226-230.

16. Johnson SM, et al. Mitigation of hematologic radiation toxicity in mice through pharmacological quiescence induced by CDK4/ 6 inhibition. J Clin Invest. 2010;120(7):2528-2536

17. Denekamp J. Cell kinetics and radiation biology. Int J Radiat Biol Relat Stud Phys Chem Med. 1986;49(2):357-380.

18. Ortega S, Malumbres M, Barbacid M. Cyclin Ddependent kinases, INK4 inhibitors and cancer. Biochim Biophys Acta. 2002;1602(1):73-87.

19. Diaz-Padilla I, Siu LL, Duran I. Cyclin-dependent kinase inhibitors as potential targeted anticancer agents. Invest New Drugs. 2009;27(6):586-594.

20. Fry DW, et al. Specific inhibition of cyclin-dependent kinase 4/ 6 by PD 0332991 and associated antitumor activity in human tumor xenografts. Mol Cancer Ther. 2004;3(11):1427-1438.

21. Demidenko ZN, Korotchkina LG, Gudkov AV, Blagosklonny MV. Paradoxical suppression of cellular senescence by p53. Proc Natl Acad Sci U S A. 2010;107(21):9660-9664.

\section{A balancing act for autophagin}

Andreas Till and Suresh Subramani

Section of Molecular Biology, Division of Biological Sciences, University of California, San Diego, La Jolla, California, USA.

\begin{abstract}
Autophagy is a tightly regulated catabolic process whereby cells degrade their constituents to dispose of unwanted cytoplasmic elements and recycle nutrients for cellular remodeling. Studies of autophagy in mammals have elicited substantial interest because it is linked to a range of physiologic and pathologic states. In this issue of the JCI, Mariño et al. uncover a role for autophagy in a balance disorder related to inner ear pathologies. Mice lacking the protease autophagy-related 4B (Atg4b, also known as autophagin-1) exhibited a systemic reduction in autophagy and showed defects in the development of otoconia, organic particles that contain calcium carbonate crystals and proteins and that are essential for balance perception (equilibrioception) in mammals. The intriguing aspect of this work is that an autophagy block impairs the secretion and assembly of otoconial proteins, emphasizing a role for autophagy in functions distinct from macromolecule degradation.
\end{abstract}

\section{The physiological importance of autophagy}

Autophagy, a tightly regulated process by which cells consume unwanted cytoplasmic macromolecular constituents and recycle nutrients for cellular remodeling, is mediated by the coordinated activity of autophagy-specific $(A T G)$ genes. There are several forms of autophagy, but here we focus on the best-characterized form, macroautophagy (referred to herein as "autophagy"). During this evolutionarily conserved process, a double membrane

Conflict of interest: The authors have declared that no conflict of interest exists.

Citation for this article: $J$ Clin Invest. 2010; 120(7):2273-2276. doi:10.1172/JCI43238. known as the isolation membrane wraps around portions of the cytoplasm to form a double-membrane vesicle, the autophagosome. The engulfed cargo, including organelles, is degraded upon autophagosome fusion with late endosomes or lysosomes.

Autophagy plays a central role in cancer, neurodegeneration, innate immunity, organelle clearance, the organismal response to starvation, and aging (1). Genetic variations in the autophagy-related genes autophagyrelated 16-like 1 (ATG16L1) and immunityrelated GTPase family $\mathrm{M}(I R G M)$ are linked to susceptibility to Crohn disease, a human chronic inflammatory disorder affecting the gastrointestinal tract $(2,3)$. Mice exhibit basal and induced forms of autophagy, both of which play important physiological roles
$(4,5)$. The former is involved in the homeostasis of cellular constituents, including organelles, and the degradation of long-lived proteins and protein aggregates $(4,5)$. However, autophagy can also be induced by physiologic and pathologic conditions, such as nutrient starvation and infection by pathogens $(4,5)$. Consequently, it should not be surprising that mice incapable of autophagy die perinatally, revealing the necessity of autophagy in surviving neonatal starvation (i.e., after the transplacental nutrient supply is stopped, autophagy becomes essential for maintaining the amino acid supply; ref. 6). Tissue-specific knockouts of autophagy genes have also revealed diverse pathologies. These include severe hepatomegaly and hepatic hypertrophy caused by liverspecific deficiency of autophagy-related 7 (Atg7) (5); behavioral problems, as indicated by abnormal limb clasping, reduced coordinated movement, and neuronal loss, caused by either Atg5 or Atg7 deficiency in the central nervous system (7); the degeneration of islet cells, reduced glucose tolerance, and insulin secretion caused by Atg7 deficiency in pancreatic $\beta$ cells $(8,9)$; and the abnormal morphology and function of intestinal Paneth cells caused by deficiency in either Atg1611 or Atg5 (10). These studies demonstrate that the physiological phenotypes are caused not only by the loss of 